\title{
Supporting Information: Mechanism of
}

\section{pKID/KIX Association Studied by Molecular Dynamics Free Energy Simulations}

\author{
Rainer Bomblies, Manuel P. Luitz, and Martin Zacharias* \\ Physik-Department T38, Technische Universität München, James-Franck-Str. 1, 85748 \\ Garching, Germany \\ E-mail: zacharias@tum.de
}

Phone: +49 89289 12335. Fax: +498928912444

${ }^{*}$ To whom correspondence should be addressed 


\section{Supporting information}

\section{Simulation of the complex}

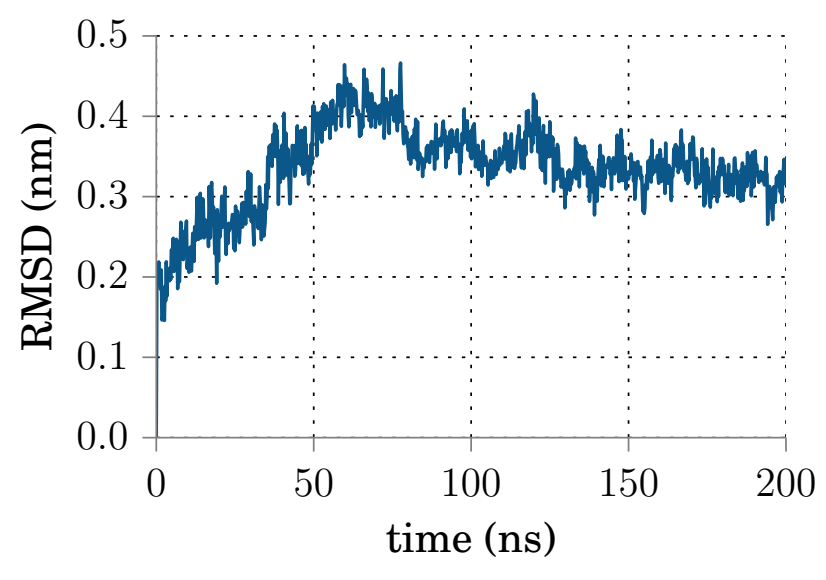

Figure S1: Backbone RMSD of pKID-KIX complex simulation relative to the NMR structure (pdb1KDX, model 1) during MD simulation. Deviations mostly arise from a slight reorganization of helix $\alpha_{A}$.

\section{Free energies obtained from FEP/BAR simulations}

Table S1: Data of free energy perturbation simulations and difference of PoissonBoltzmann-calculations for salt concentrations $50 \mathrm{mM}$ to $10 \mathrm{mM}$. All values in $\mathrm{kJ} / \mathrm{mol}$.

\begin{tabular}{l|ccccccc} 
& $\Delta \Delta G$ & ITC $^{\mathrm{S} 1}$ & ITC $2^{\mathrm{S} 2}$ & decharge & vdw & recharge & PB \\
\hline Y650A & 5.62 & 5.90 & 6.30 & & & & \\
Y658F & 10.24 & 10.20 & 8.90 & & & & \\
K662A & 9.98 & 6.20 & 4.70 & 9.74 & 0.10 & 0.14 & -0.62 \\
R124A & 10.83 & & & 11.89 & -1.05 & -0.01 & -0.84 \\
L128A & 11.16 & & & & & & \\
R131A & 6.24 & & & 6.03 & 0.36 & -0.15 & -1.03 \\
pS133S & 10.51 & & 9.10 & 10.00 & 2.91 & -2.40 & 1.51 \\
Y134A & 5.24 & & & & & & \\
I137A & 6.32 & & & & & & \\
L138A & 12.37 & & & & & &
\end{tabular}



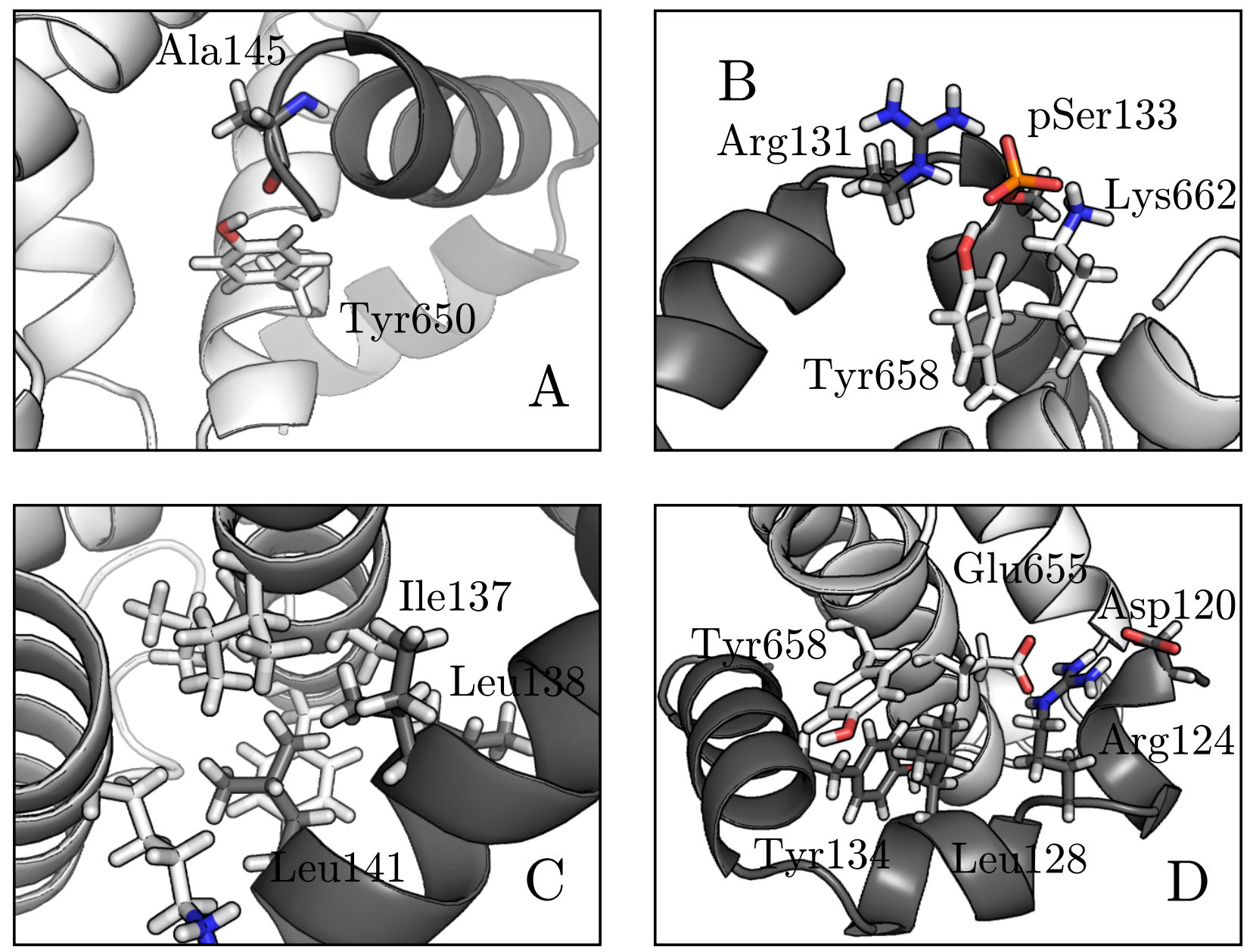

Figure S2: Snapshots from wild type replicas of the FEP-BAR simulations. Image A shows the hydrogen bond between Tyr650 and Ala145, B shows the interaction network around the phosphorylation site, C shows side chains of pKID's helix $\alpha_{B}$ in contact with the hydrophobic groove of KIX and D shows interactions of pKID's helix $\alpha_{A}$. 


\section{Continuous MD simulations}

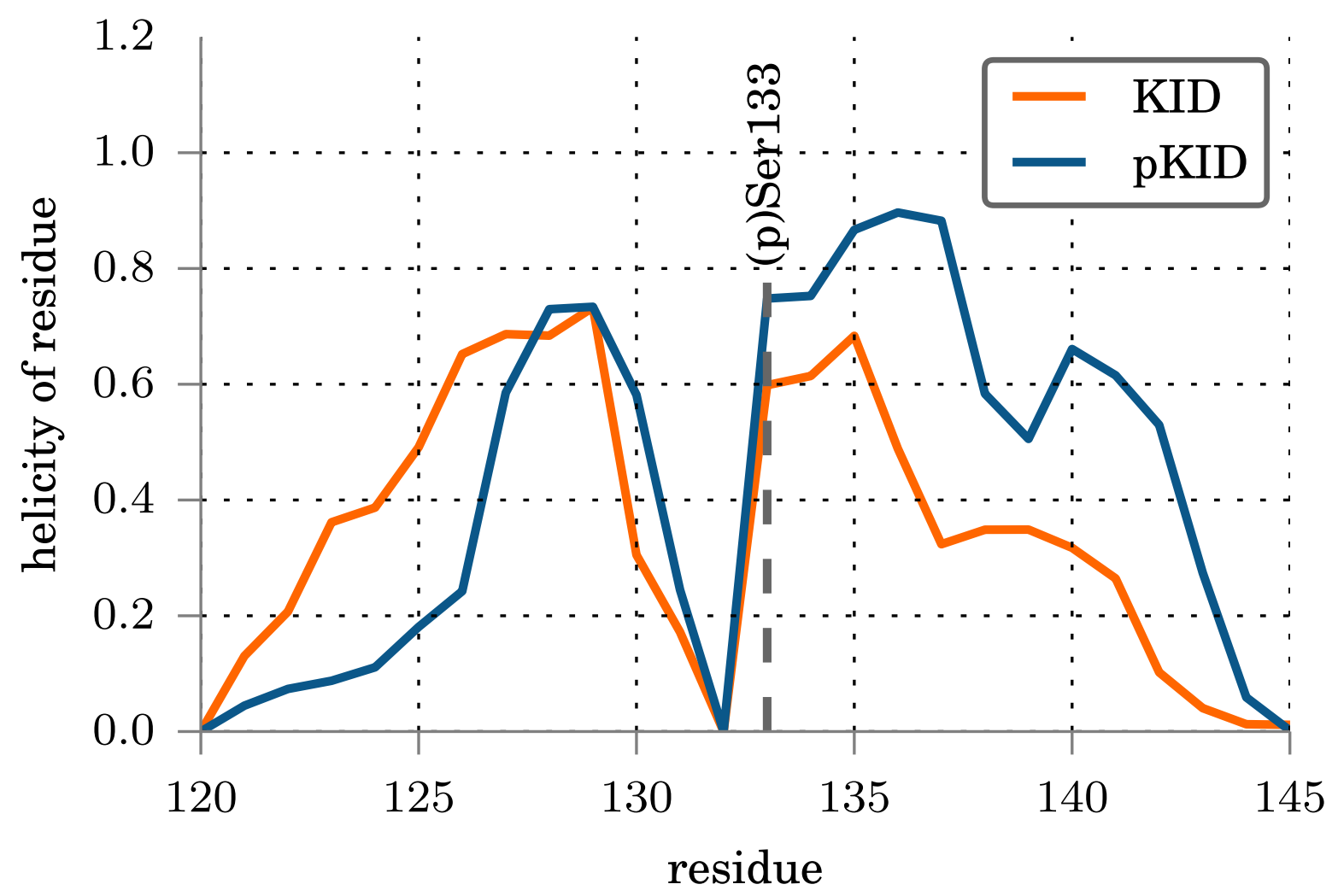

Figure S3: Mean helicity calculated with the DSSP algorithm ${ }^{\mathrm{S} 3}$ for $1 \mu \mathrm{s}$ continuous simulations of pKID and KID, respectively. 


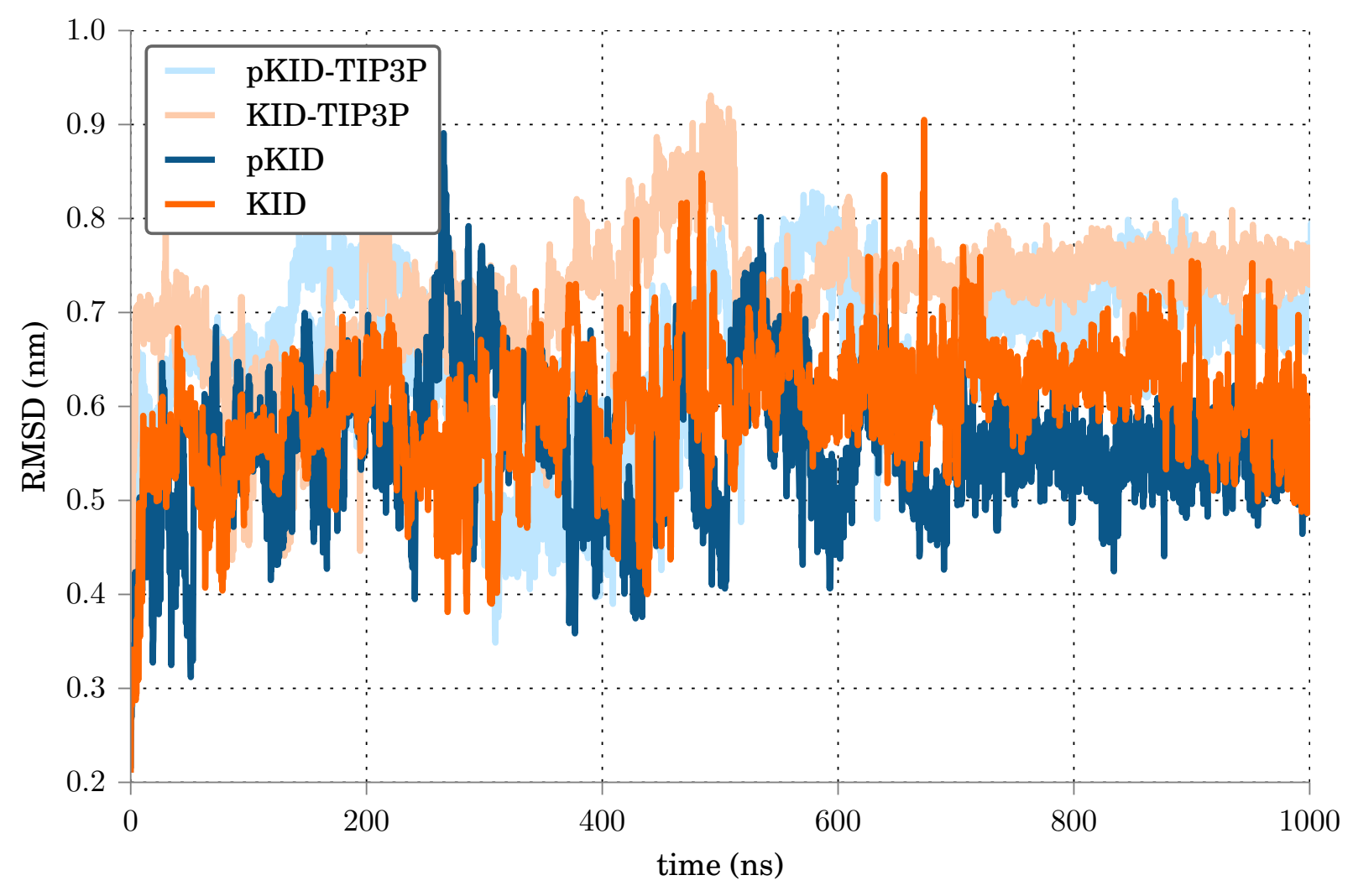

Figure S4: Backbone RMSD of continuous MD simulations with TIP3P and TIP4P-D water models. With TIP3P trapping of structures for hundreds of ns, e.g. for KID from $600 \mathrm{~ns}$ to 1000 ns is observed. 


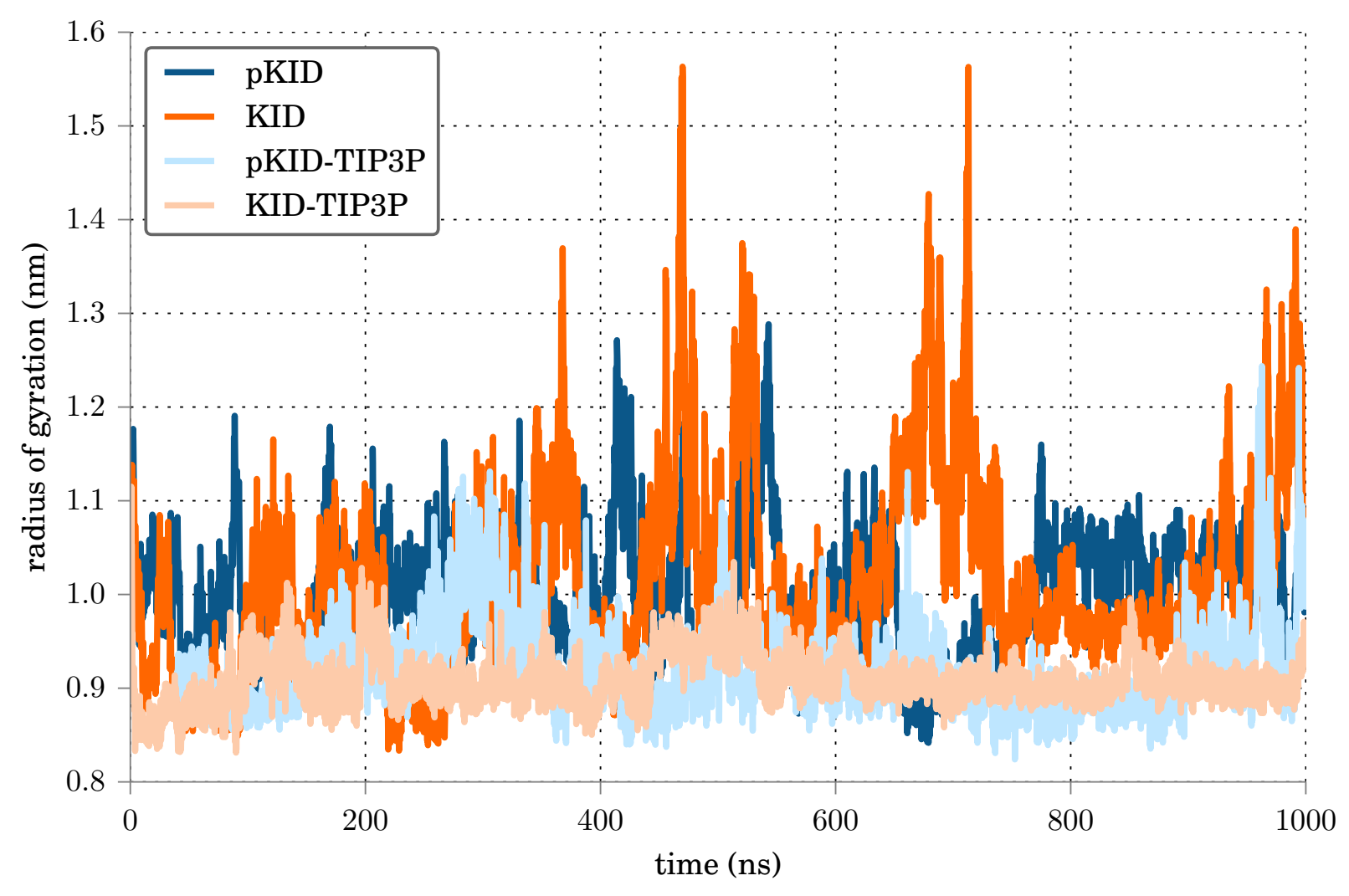

Figure S5: Radius of gyration of continuous MD simulations with TIP3P and TIP4P-D water models. With TIP3P more collapsed states are sampled. 


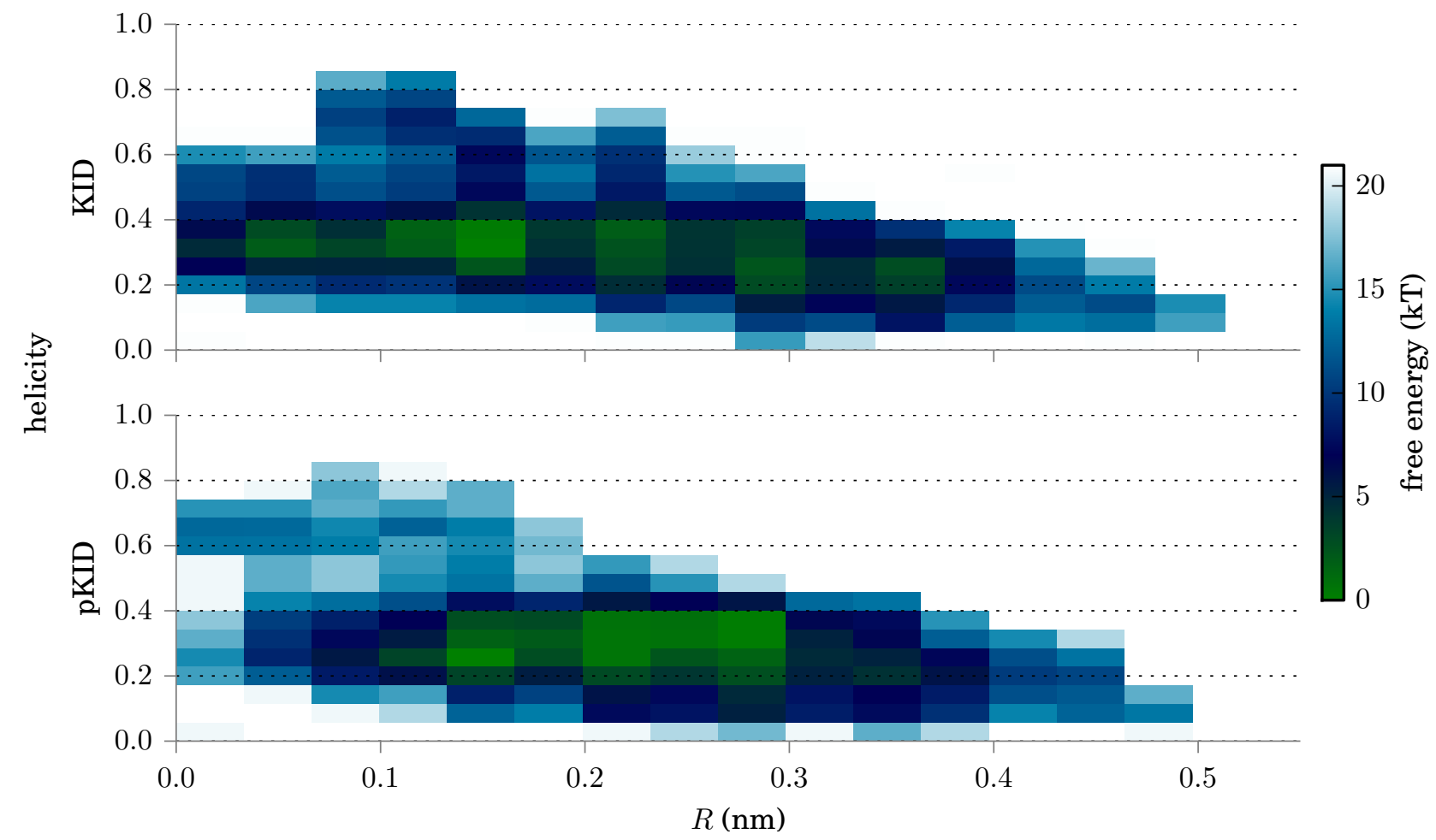

Figure S6: Free energy surface in the space of helicity and dRMSD reaction coordinate $R$ (as defined for the core motif) for continuous MD simulations of and KID and pKID. The main free energy basin of pKID is narrower and at slightly higher helical content. KID structures are distributed over a wider area of $R$ but also reach higher helical content more frequently. 


\section{Advanced sampling along the dRMSD reaction coordinate}

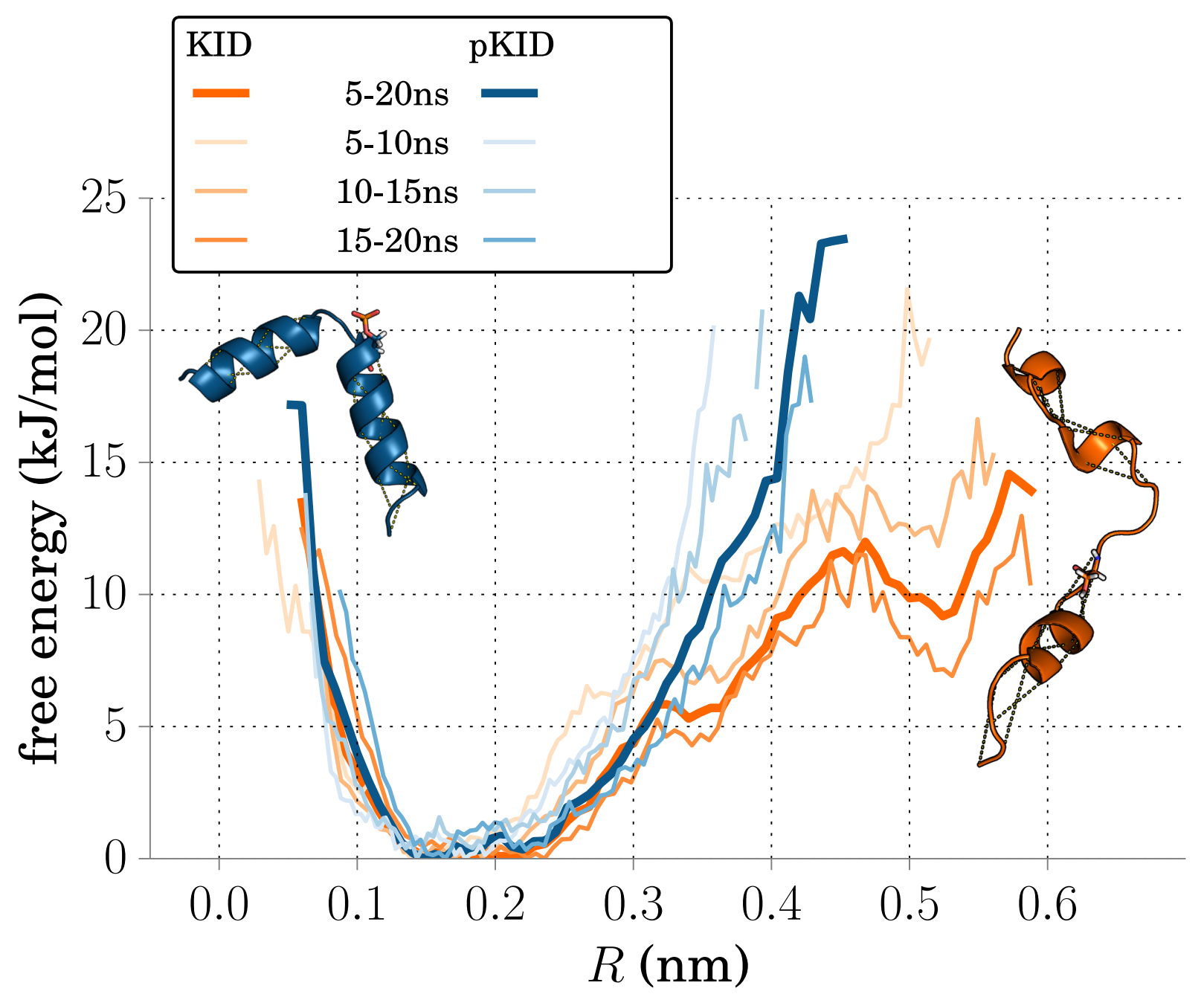

Figure S7: Calculated PMF of the full KID protein unfolding/folding along the $R$ coordinate where low dRMSD indicates close resemblance to the folded structure and high values correspond to unfolded structures. Thick lines indicate the total PMF after 5 ns equilibration, thinner lines show PMFs of simulation time intervals of the simulation. Full (p)KID, as shown in the structure inset, was simulated with all pairs contributing to $R$ indicated by yellow dashed lines. 


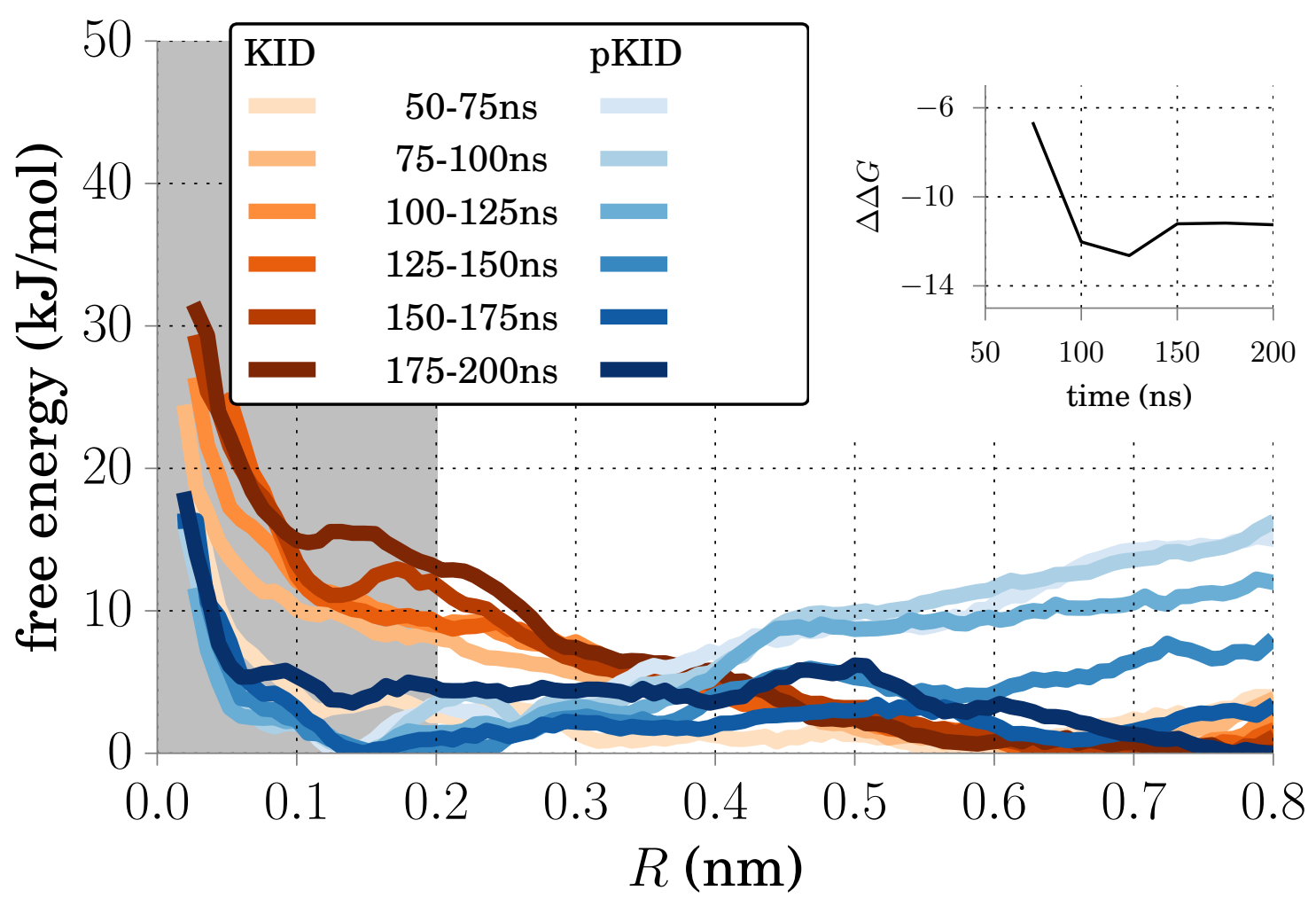

Figure S8: Calculated PMF obtained from different time intervals of the US simulations along the dRMSD coordinate of the kinked core motif. Inset shows the difference of free energy gain upon folding: $\Delta G_{\text {fold }}(\mathrm{KID})-\Delta G_{\text {fold }}(\mathrm{pKID})$. For the different time intervals the calculated free energy difference between pKID and KID remains approximately constant. 


\section{Simulation of the pKID folding process after encounter binding at}

\section{the pSer133 binding site}

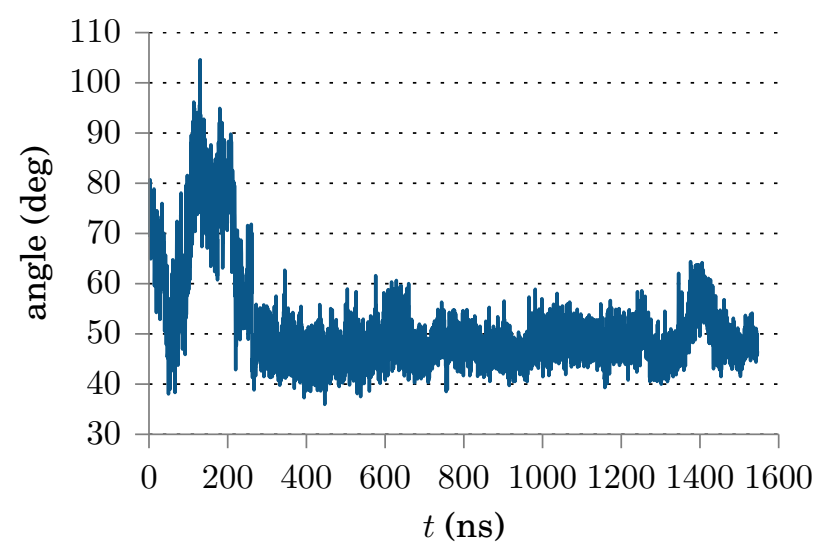

Figure S9: Angle between $C_{\alpha}$ atoms of residues 125, 131 and 137 sampled during the successful association simulation of pKID to KIX. The correct folding of helix $\alpha_{A}$ (at $200 \mathrm{~ns}$ ) and the first helical turn of helix $\alpha_{B}$ (after $240 \mathrm{ns)} \mathrm{is} \mathrm{coupled} \mathrm{to} \mathrm{a} \mathrm{drop} \mathrm{of} \mathrm{the} \mathrm{kink} \mathrm{angle.}$ 


\section{References}

[S1] Parker, D.; Ferreri, K.; Nakajima, T.; LaMorte, V.; Evans, R.; Koerber, S.; Hoeger, C.; Montminy, M. Phosphorylation of CREB at Ser-133 Induces Complex Formation With CREB-binding Protein via a Direct Mechanism. Mol. Cell. Biol. 1996, 16, 694-703.

[S2] Zor, T.; Mayr, B. M.; Dyson, H. J.; Montminy, M. R.; Wright, P. E. Roles of Phosphorylation and Helix Propensity in the Binding of the KIX Domain of CREB-binding Protein by Constitutive (c-Myb) and Inducible (CREB) Activators. J. Biol. Chem. 2002, 277, 42241-42248.

[S3] Kabsch, W.; Sander, C. Dictionary of Protein Secondary Structure: Pattern Recognition of Hydrogen-bonded and Geometrical Features. Biopolymers 1983, 22, 2577-2637. 